\title{
Topological Design of Compliant Mechanisms with Multiple Materials
}

\author{
Jinqing Zhan ${ }^{1,2,3}$, Liangming Long ${ }^{2}$, Chunhui Lin $^{2}$ and Xiangjie Yang ${ }^{1,3 *}$ \\ ${ }^{1}$ Jiangxi Xiongying Aluminum Co., Ltd, Nanchang 330500, China \\ ${ }^{2}$ School of Mechanotronics and Vehicle Engineering, East China Jiaotong University, Nanchang 330013, China \\ ${ }^{3}$ School of Mechanical and Electrical Engineering, Nanchang University, Nanchang 330031, China \\ ${ }^{*}$ Corresponding author
}

\begin{abstract}
In order to obtain better mechanical properties by exploiting the advantages of different materials, a topological design method of compliant mechanisms multiple materials was proposed. The multiple materials interpolation model for topological design of compliant mechanisms was established using the generalized Solid Isotropic Material with Penalization method. The objective function was developed maximizing the output displacement, and the structural volume was restricted. The adjoint approach is applied to perform the sensitivity analysis. The improved filter technique was applied to modify the sensitivities in order to avoid the avoid the numerical instability phenomenon. The method of moving asymptotes was used to solve the topological optimization problem. The numerical examples were given to illustrate that the proposed method is correct and effective. The result shows that multiple materials compliant mechanisms has better mechanical performance than single-phase material compliant mechanisms.
\end{abstract}

Keywords-topology optimization; compliant mechanisms; multiple materials; the improved filter technique

\section{INTRODUCTION}

Compliant mechanisms are flexible monolithic structures that can achieve force and motion transmission through elastic deformation of relative flexibility of its members[1]. Compared with rigid-body mechanisms, compliant mechanisms have many advantages such as high precision, ease of fabrication, easy integration, no friction losses and without any assembly cost. Thus, it has been applied widely in the micro-electromechanical systems, precision positioning, micro/nano manufacturing, fiber alignment and aerospace[2]. Topological design of compliant mechanisms has drawn more and more attentions because it only needs to designate a design domain and the positions of the inputs and outputs.

In recent years, the research on the topology optimization of compliant mechanisms has been greatly developed[3-5]. Topological design of compliant mechanisms with a single material has been unable to meet certain requirements. However, multiple materials compliant mechanisms has better mechanical performance exploiting the advantages of different materials. Sigmund[6] proposed a topology optimization method to design electro-thermo-mechanical actuators using two different materials. Yin et al.[7] used a peak function method to design multi-material compliant mechanisms. Alonso et al.[8] adopted the sequential element rejection and admission method to produce compliant mechanism with multiple materials. Gaynor et al.[9] proposed a topological design and fabrication process for multiple materials compliant structures. Zhang et al.[10] developed a new parallel strategy method for multi-objective topology optimization of multiple materials compliant mechanisms. Compared to the vast amount of literature on a single material compliant mechanisms, study on topological design of compliant mechanisms with multiple materials are relatively few.

In this study, we proposed a topological design method of multiple materials compliant mechanisms using the improved filter technique. The multiple materials interpolation model for topological design of compliant mechanisms was established using the generalized Solid Isotropic Material with Penalization model. The objective function was developed maximizing the output displacement, and the structural volume was restricted. The adjoint approach is applied to perform the design sensitivity analysis. The modified filter technique was applied to modify the sensitivities in order to avoid the avoid the phenomenon of numerical instability. The method of moving asymptotes was used to solve the topological optimization problem.

\section{OPTIMIZATION Formulations OF MUlTiPle MATERIALS COMPLIANT MECHANISMS}

\section{A Multiple Materials Interpolation Scheme}

The material interpolation model for topology optimization is mainly homogenization method ${ }^{[11]}$ and Solid isotropic material with penalization (SIMP) method ${ }^{[12]}$. Compared with the homogenization method, the Solid isotropic material with penalization method has fewer design variables, and is easy to calculate. In this paper, SIMP method is adopted as material interpolation method to establish the topology optimization model with multiphase material.

Based on the generalized SIMP interpolation mode ${ }^{[12]}$, the three-phase material interpolation model of elastic modulus for each element can be expressed as

$$
\begin{aligned}
& E_{i, j}\left(x_{1}^{e}, x_{2}^{e}\right)=\left(x_{1}^{e}\right)^{p_{1}}\left[\left(x_{2}^{e}\right)^{p_{2}} E_{1}\right. \\
& \left.\quad+\left(1-\left(x_{2}^{e}\right)^{p_{2}}\right) E_{2}\right]+\left(1-\left(x_{1}^{e}\right)^{p_{1}}\right) E_{\text {void }}
\end{aligned}
$$


Where, the first set of design variables $x_{1}$ are used in determining the optimal topology, and the second set of design variables $x_{2}$ are used for selecting the material at each location within the topology. $E_{1}, E_{2}$ and $E_{\text {void }}$ are the elasticity modulus of the solid material 1 , the solid material 2 and the void material, respectively. $p_{1}$ and $p_{2}$ are the penalty coefficients, and are taken as 3 .

Each phase material is decided by the combination of two set of design variables.

$$
\left(x_{1}^{e}, x_{2}^{e}\right)= \begin{cases}(1,0) & \text { solid phase } 1 \\ (1,1) & \text { solid phase } 2 \\ (0,0) & \text { void }\end{cases}
$$

The distribution of three-phase material is shown in Fig.1

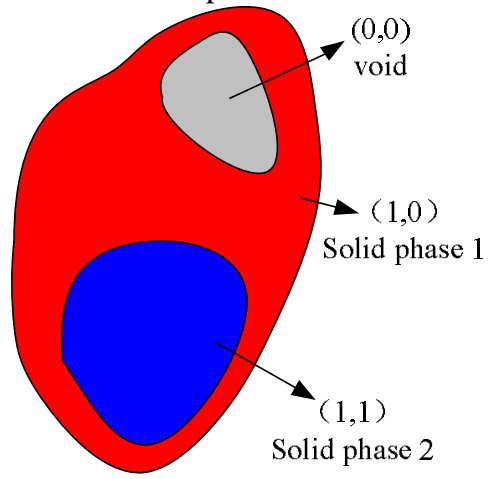

FIGURE I. DISTRIBUTION OF EACH PHASE MATERIAL

\section{B Optimization Model for Multiple Materials Compliant Mechanisms}

The flexibility requirement of compliant mechanisms is determined by maximizing the output displacement at the output point. The output displacement can be defined using the unit dummy load method. A unit dummy load $\mathbf{F}_{1}$ are applied at the output point in the desired direction, as shown in Fig. 2. The output displacement can be defined as

$$
U_{\text {out }}=\mathbf{F}_{1} \mathbf{U}
$$

where $\mathbf{U}$ is the nodal displacement vector.

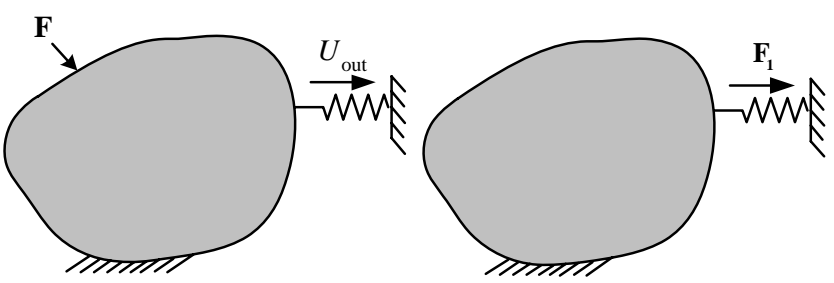

FIGURE II. DESIGN DOMAIN FOR MULTIPLE MATERIALS COMPLIANT MECHANISM
The objective of the optimization problem is to maximize the output displacement, and each phase material volume was restricted is used as the constraints. The optimization model can be established as

$$
\begin{aligned}
& \text { Maximize : } U_{\text {out }}\left(x_{1}, x_{2}\right)=\mathbf{F}_{1} \mathbf{U} \\
& \text { Subject to }: \begin{cases}\mathbf{K U}=\mathbf{F} \\
V_{1}\left(x_{1}, x_{2}\right)=\sum_{e=1}^{N} x_{1}^{e} x_{2}^{e} V^{e} \leq V_{1}^{*} \\
V_{2}\left(x_{1}, x_{2}\right)=\sum_{e=1}^{N} x_{1}^{e}\left(1-x_{2}^{e}\right) V^{e} \leq V_{2}^{*} \\
0 \leq x_{1}^{e} \leq 1 \quad(e=1,2, \cdots, N) \\
0 \leq x_{2}^{e} \leq 1 \quad(e=1,2, \cdots, N)\end{cases}
\end{aligned}
$$

Where, $\mathbf{F}$ refers to the input load vector, $\mathbf{K}$ is the global stiffness matrix, $N$ represents the total number of finite elements. $V_{1}^{*}$ and $V_{2}^{*}$ are the allowable volume of the solid material 1 and those of the solid material 2, respectively. $V^{e}$ is the solid element volume.

\section{Sensitivity Analysis and Solution Technique}

To apply the gradient-based algorithm, the sensitivities of the objective and constraint with respect to the design variables are need to solved. The derivative of the output displacement with respect to the design variables is stated as

$$
\frac{\partial U_{\text {out }}}{\partial x_{1}^{e}}=\mathbf{F}_{1} \frac{\partial \mathbf{U}}{\partial x_{1}^{e}}
$$

The adjoint method is adopted to solve the term $\frac{\partial \mathbf{U}}{\partial x_{1}^{e}}$ in "(5)". It is obtained form the global equilibrium equation

$$
\frac{\partial \mathbf{K}}{\partial x_{1}^{e}} \mathbf{U}+\mathbf{K} \frac{\partial \mathbf{U}}{\partial x_{1}^{e}}=0
$$

Thus, the term $\frac{\partial \mathbf{U}}{\partial x_{1}^{e}}$ can be calculated

$$
\frac{\partial U_{\text {out }}}{\partial x_{1}^{e}}=-\mathbf{K}^{-1} \frac{\partial \mathbf{K}_{e}}{\partial x_{1}^{e}} \mathbf{U}
$$

Considering the unit dummy load, we have

$$
\mathrm{KU}_{1}=\mathbf{F}_{1}
$$

where $\mathbf{U}_{1}$ is the nodal displacement vector under the unit dummy load. 
Substituting "(7)" and "(8)" into "(5)", we finally obtain the sensitivity as

$$
\frac{\partial U_{\text {out }}}{\partial x_{2}^{e}}=-\mathbf{U}_{1}^{\mathrm{T}} \frac{\partial \mathbf{K}_{e}}{\partial x_{2}^{e}} \mathbf{U}
$$

Similarly, we can get

$$
\frac{\partial U_{\text {out }}}{\partial x_{2}^{e}}=-\mathbf{U}_{1}^{\mathrm{T}} \frac{\partial \mathbf{K}_{e}}{\partial x_{2}^{e}} \mathbf{U}
$$

Where

$$
\begin{gathered}
\frac{\partial \mathbf{K}_{e}}{\partial x_{1}^{e}}=p_{1}\left(x_{1}^{e}\right)^{p_{1}-1}\left[\left(x_{2}^{e}\right)^{p_{2}} E_{1}\right. \\
\left.+\left(1-\left(x_{2}^{e}\right)^{p_{2}}\right) E_{2}-E_{\text {void }}\right] \cdot \mathbf{K}_{g e} \\
\frac{\partial \mathbf{K}_{e}}{\partial x_{2}^{e}}=\left(x_{1}^{e}\right)^{p_{1}} p_{2}\left(x_{2}^{e}\right)^{p_{2}-1}\left(E_{1}-E_{2}\right) \cdot \mathbf{K}_{g e}
\end{gathered}
$$

Where $\mathbf{K}_{e}$ is the element stiffness matrix, $\mathbf{K}_{g e}$ refers to the product of the element stiffness matrix and the reciprocal of the element elasticity modulus.

The optimization problems are solved usually by two approaches: Optimality Criteria (OC) method [13] and mathematical programming method. The OC method is simple and easy to be implemented in topological design. But, it is not suitable for multi-constrained topology optimization problem. In mathematical programming method, sequential convex programming approaches include the Method of Moving Asymptotes (MMA) [14], Sequential linear programming (SLP)[11] and others. MMA is more flexible to deal with multi-constrained optimization problem. MMA is adopted to update the design variables in the optimization problem.

\section{$D$ The Improved Filtering Technology}

The designs of multiple materials compliant mechanisms using topology optimization typically lead to checkerboard patterns. In this paper, the improved filter technology[15] is adopted to modify the sensitivities of the objective and the constraints for eliminating the numerical instability phenomena.

The sensitivity of the objective function is modified as

$$
\frac{\partial \hat{U}_{\text {out }}}{\partial x_{i}^{e}}=\frac{\sum_{f=1}^{N_{e}} H_{e f} \frac{\partial U_{\text {out }}}{\partial x_{i}^{f}}}{\sum_{f=1}^{N_{e}} H_{e f}}
$$

Where, $\frac{\partial \hat{U}_{\text {out }}}{\partial x_{i}^{e}}$ represents the modified sensitivity of the output displacement with respect to $i$ th $(i=1,2)$ design variable for element $e, \frac{\partial U_{\text {out }}}{\partial x_{i}^{e}}$ is the sensitivity of the output displacement with respect to $i$ th design variable for element $f . N_{e}$ is the set of element $e$ for which the distance to element is smaller than the filter radius $r_{\min }, H_{e f}$ is the weight factor.

The weight factor is defined as

$$
H_{e f}=\max \left(0, r_{\min }-\operatorname{dist}(e, f)\right)
$$

Where, $\operatorname{dist}(e, f)$ refers to the center-to-center distant between element $e$ and element $f$.

In "(7)", the sensitivity of the objective function is modified by the weighted average in the sub-domain where the center-tocenter distance is smaller than the filter radius. It can not eliminate thoroughly the checkerboard patterns. Therefore, the density filter is adopted to transforms the original densities as follows

$$
\hat{x}_{i}^{e}=\frac{1}{\sum_{f=1}^{N_{e}} H_{e f}} \sum_{f=1}^{N_{e}} H_{e f} x_{i}^{f}
$$

Where, the filtered density $\hat{x}_{i}^{e}$ for element $e$ refers to the physical density. $x_{i}^{f}$ is $i$ th design variable design variable for element $f$.

When density filter is applied, the sensitivities of the objective and the constraint with respect to the design variables are obtained by

$$
\frac{\partial \psi}{\partial x_{i}^{e}}=\sum_{f=1}^{N_{e}} \frac{1}{\sum_{f=1}^{N_{e}} H_{e f}} H_{e f} \frac{\partial \psi}{\partial \hat{x}_{i}^{e}}
$$

Where $\psi$ represents the objective or the constraint.

While the filtering technique is adopted, the value of the filter radius has the influence on the configuration of compliant mechanisms. In this paper, the optimization strategy of filter radius is used to choose the optimal value during the optimization process[15].The large value of filter radius is applied in the initial stage of the optimization. If the filter radius is larger than the set value, the value of the filter radius for the current iteration step is changed to 0.99 times the value for the last iteration step. The value of the filter radius is reduced gradually up to convergence. 


\section{NUMERICAL EXAMPLES}

In this section, we present some numerical examples of topological design of multiple materials compliant gripper. The design domain, boundary conditions, and the prescribed output motion are shown in Fig. 3. The size of the design domain is 80 $\mathrm{mm}$ by $80 \mathrm{~mm}$. we use Young's Modulus 1 for the solid material $1=2 \mathrm{MPa}$, Young's Modulus 2 for the solid material 2 $=1 \mathrm{MPa}$, allowable volume $1=10 \%$, allowable volume $2=$ $20 \%$, Poisson's Ratio $=0.3$. The output springs stiffness is set to $k_{\text {out }}=1 \mathrm{~N} / \mathrm{mm}$. Applying symmetry, the lower half of the design domain contains 3200 elements.

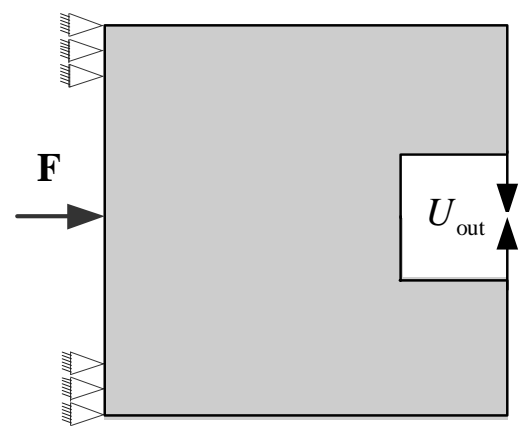

FIGURE III. DESIGN DOMAIN OF COMPLIANT GRIPPER

In order to compare topological result of single-phase material with that of multiple materials, topological design of compliant mechanisms with single-phase material is carried out. we use Young's modulus is E $=1 \mathrm{MPa}$, Poisson's ratio $\mu=$ 0.3 , allowable volume $=30 \%$ while other parameters keep the same.

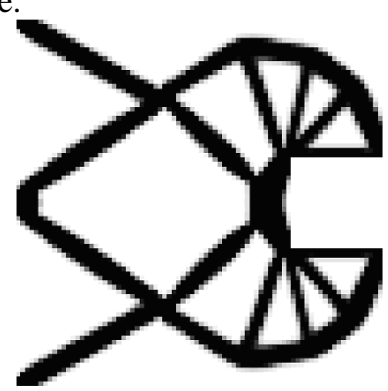

(A)

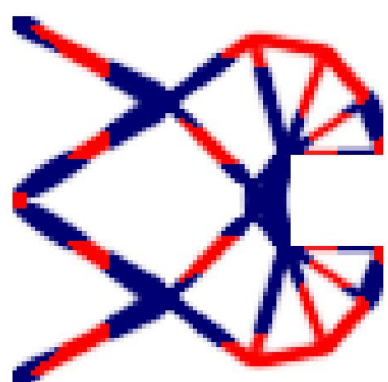

(B)
FIGURE IV. COMPLIANT GRIPPERS OBTAINED BY DIFFERENT METHODS: (A) SINGLE-PHASE MATERIAL TOPOLOGY OPTIMIZATION (B) MULTIPLE MATERIALS TOPOLOGY OPTIMIZATION

Single-phase material and multiple materials compliant gripper are obtained by topology optimization as shown in Fig. 4(a) and Fig. 4(b), respectively. In Fig. 4(b), the red area represents the stiff material, the blue region refers to the soft material, and the white area is void. The output displacement of the optimal mechanism obtained by single-phase material is $11.5 \mathrm{~mm}$, and that of the optimal mechanism obtained by multiple materials is $14.2 \mathrm{~mm}$. The output displacement is increased by $23.4 \%$ using multiple materials topology optimization. It shows that the multiple materials topology optimization has better mechanical performance. That is because the stiff material is largely distributed in the region of small deformation, and the soft material is largely distributed in the region of large deformation. In addition, the configuration of multiple materials compliant gripper is similar to that of single-phase material compliant gripper. It can shows that the proposed method is correct.

In order to investigate the influence of the ratio of the elastic modulus on the obtained mechanical properties. the ratio of the elastic modulus is set to $1.5: 1,2: 1,2.5: 1$, and $3: 1$ while other parameters keep the same. The Young's Modulus 2 for the solid material 2 is set to $1 \mathrm{MPa}$ while the Young's Modulus 1 is changed.

Fig.5 shows topological configuration of multiple materials mechanism gripper for different ratio of the elastic modulus. From Table 1, we can find that the output displacement of the obtained mechanism gripper is larger by increasing the ratio of the elastic modulus. Thus, multiple materials compliant gripper has better mechanical performance increasing the ratio of the elasticity modulus within a certain range.

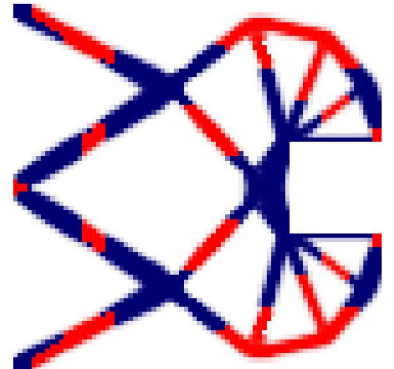

(A)

(C)

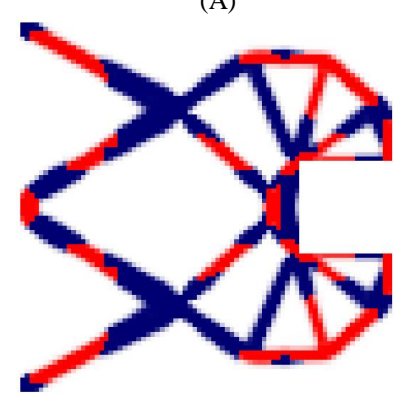

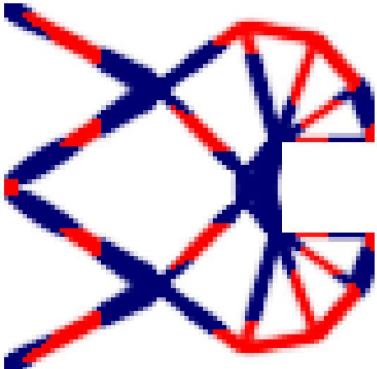

(B)

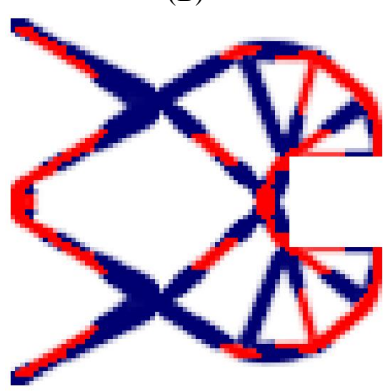

(D)
FIGURE V. COMPLIANT GRIPPERS FOR DIFFERENT RATIO OF ELASTIC MODULUS: (A) $E_{1}: E_{2}=1.5: 1$ (B) $E_{1}: E_{2}=2: 1$ (C) $E_{1}: E_{2}=2.5: 1(\mathrm{D}) E_{1}: E_{2}=3: 1$

TABLE I. OUTPUT DISPLACEMENT OF COMPLIANT GRIPPER FOR DIFFERENT RATIO OF ELASTIC MODULUS

\begin{tabular}{|c|c|}
\hline $\begin{array}{c}\text { Ratio of Elastic Modulus } \\
E_{1}: E_{2}\end{array}$ & $\begin{array}{c}\text { Output Displacement } \\
U_{\text {out }}(\mu \mathrm{m})\end{array}$ \\
\hline $1.5: 1$ & 13.6 \\
\hline $2: 1$ & 14.2 \\
\hline $2.5: 1$ & 16.2 \\
\hline $3: 1$ & 21.9 \\
\hline
\end{tabular}




\section{CONCLUSIONS}

A new method for topology optimization of multiple materials compliant mechanisms by the improved filter technique is presented. The topology optimization model of multiple materials compliant mechanisms is established using the generalized Solid Isotropic Material with Penalization model. The improved filter technique is applied to modify the sensitivities of the objective and the constraint. Compared with single-phase material topology optimization, the multiple materials compliant mechanisms has better mechanical performance because the stiff material is largely distributed in the region of small deformation and the soft material is largely distributed in the region of large deformation. In addition, multiple materials compliant gripper has better mechanical performance increasing the ratio of the elasticity modulus within a certain range.

\section{ACKNOWLEDGMENT}

This research was supported by the National Natural Science Foundation of China (51305136), the Natural Science Foundation of Jiangxi, China (20151BAB206032, 20161BAB206152) and the Science and Technology Plan Projects of Jiangxi Provincial Education Department (GJJ150547). This supports are greatly acknowledged.

\section{REFERENCES}

[1] L.L.Howell, Compliant mechanisms, John Wiley \& Sons: New York, 2001 ,

[2] O. Sigmund, "On the design of compliant mechanisms using topology optimization," Mechanics of Structures and Machines, vol. 25, pp. 495526, April 1997.

[3] J.Z. Lin, Z. Luo, L. Y. Tong, "A new multi-objective programming scheme for topology optimization of compliant mechanisms," Structural Multidisciplinary Optimization, vol. 40, pp. 241-255, January 2010.

[4] Y. X. Du, Z. Luo, Q. H. Tian, et al. "Topology optimization for thermomechanical compliant actuators using mesh-free methods," Engineering Optimization, vol. 41, pp. 753-772, July 2009.

[5] L. Mello, R. Salas, E. Silva. "On response time reduction of electrothermomechanical MEMS using topology optimization," Computer Methods in Applied Mechanics and Engineering, vol. 247248, pp. 93-102, November 2012.

[6] O.Sigmund, "Design of multiphysics actuators using topology optimization part II: two-material structures," Computer Methods in Applied Mechanics and Engineering, vol. 190, pp. 6605-6627, October 2001.

[7] L. Yin, G. K.Ananthasuresh, "Topology optimization of compliant mechanisms with multiple materials using a peak function material interpolation scheme," Structural \& Multidisciplinary Optimization, vol. 23, pp. 49-62, December 2001.

[8] C. Alonso, R. Ansola, O. M. Querin, "Topology synthesis of multimaterial compliant mechanisms with a Sequential Element Rejection and Admission method," Finite Elements in Analysis \& Design, vol. 85, pp. 11-19, August 2014.

[9] A. T. Gaynor, N. A. Meisel, C. B. Williams, et al. "Multiple-material topology optimization of compliant mechanisms created via PolyJet three-dimensional printing," Journal of Manufacturing Science and Engineering, vol. 136, pp. 061015(1-10), October 2014.

[10] X. M. Zhang, K. Hu, N. F. Wang, et al. "Multi-objective topology optimization of multiple materials compliant mechanisms based on parallel strategy," Journal of Mechanical Engineering, vol. 52, pp. 1-8, October 2016.(In Chinese)

[11] S. Nishiwaki, M. I. Frecher, S. MIN, N. Kikichi, "Topology optimization of compliant mechanisms using the homogenization method,"
International Journal for Numerical Methods in Engineering, vol. 42, pp. 535--559, June 1998

[12] M. P. Bendsoe, O. Sigmund, "Material interpolation schemes in topology optimization," Archive of Mechanics, vol. 69, pp. 635-654, May 1999

[13] M. Zhou, G. Rozvany, "The COC algorithm, part II: topological, geometry and generalized shape optimization," Computer Methods in Applied Mechanics \& Engineering, vol. 89, pp. 309-336, August 1991

[14] K. Svanberg, "The method of moving asymptotes: a new method for structural optimization," International Journal for Numerical Methods in Engineering, vol. 42, pp. 359-373, February 1987

[15] K. Long, Z. X. Zuo, "Topology Optimization for Compliant Mechanism Using Material Point Topological Variable," Journal of Computer-Aided Design \& Computer Graphics, vol. 22, pp. 000158-164, January 2010. 